\title{
Evolución y tendencias investigativas en marketing orientado a industrias culturales y creativas: un análisis bibliométrico en Scopus*
}

\author{
Recibido: 11 de diciembre de 2019 • Aprobado: 23 de diciembre de 2019 \\ https://doi.org/10.22395//seec.v22n53a8 \\ Jackeline Valencia** $^{* *}$ \\ Ana María Umba López ${ }^{* *}$ \\ Ana María Moná**** \\ Alejandro Valencia Arias \\ Camilo Patiño Vanegas ${ }^{* * * * * *}$
}

\begin{abstract}
RESUMEN
El objetivo de esta investigación es examinar la evolución y tendencias investigativas sobre marketing orientadas a industrias culturales y creativas. La metodología parte de un análisis bibliométrico de 374 documentos académicos obtenidos a partir de una ecuación de búsqueda formulada en Scopus, para esto se plantearon indicadores de cantidad, calidad y estructura. Entre los hallazgos, se observa que es necesario un mayor enfoque en economías emergentes, ya que su contexto social, cultural y económico es particular. Se concluye que los temas más investigados son: estrategia de mercado, diseño de producto, influencia del factor cultural, gestión de la industria (en términos de capacidades operativas y operacionalización) y acciones de internacionalización orientadas a la expansión de dichas industrias.
\end{abstract}

PALABRAS CLAVE

Marketing cultural; industrias creativas; tendencias investigativas; bibliometría.

\section{CLASIFICACIÓN JEL}

C89, D83

\section{CONTENIDO}

Introducción; 1. Metodología; 2. Resultados; Conclusiones; Referencias.

Esta investigación hace parte del trabajo interdisciplinar entre los grupos de investigación en Artes y Humanidades del ITM (Categoría A en Colciencias), Games (Categoría C en Colciencias) y el Semillero de Investigación Aneiap de la Universidad Nacional de Colombia. El proyecto de investigación que origina este artículo se denomina Tendencias investigativas en marketing orientado a industrias culturales y creativas. Las entidades financiadoras del artículo son: el Semillero de Investigación Aneiap de la Universidad Nacional de Colombia y el Centro de Investigaciones de la Institución Universitaria Escolme. El proyecto se ejecutó entre febrero y noviembre de 2019

. Artista visual, Instituto Tecnológico Metropolitano, Medellín, Colombia. Candidata a magíster en Gestión Cultural, Universidad de Barcelona, Barcelona, España. Investigadora del grupo Artes y Humanidades del Instituto Tecnológico Metropolitano, Medellín, Colombia. Investigadora del grupo Games, Institución Universitaria Escolme, Medellín, Colombia.Correo electrónico: jvalenar@alumnes.ub.edu; javalenca.a@gmail.com. Orcid: http://orcid. org/0000-0001-6524-9577

... Ingeniera industrial, Universidad Nacional de Colombia, Medellín, Colombia. Integrante del grupo de investigación GIGF, Institución Universitaria Escolme, Medellín, Colombia. Correo electrónico: amumbal@unal.edu.co. Orcid: http://orcid.org/0000-0003-1295-4189

.... Estudiante de Ingeniería Administrativa, Universidad Nacional de Colombia, Medellín, Colombia. Coordinadora del Semillero de Investigación Aneiap, Universidad Nacional de Colombia, Medellín, Colombia. Correo electrónico: ammonaz@unal.edu.co. Orcid: http://orcid.org/0000-0003-3987-1885

..... Ingeniero administrador y doctor en Ingeniería, Industria y Organizaciones, Universidad Nacional de Colombia, Medellín, Colombia. Docente e investigador del Grupo de Investigación Engineeri@, Corporación Universitaria Americana, Medellín, Colombia. Correo electrónico: javalenciar@gmail.com. Orcid: http://orcid.org/0000-00019434-6923

...... Ingeniero en Telecomunicaciones y magíster en Gestión de la Innovación Tecnológica, Cooperación y Desarrollo Regional, Instituto Tecnológico Metropolitano, Medellín, Colombia. Doctor en Pensamiento Complejo, Multiversidad Edgar Morin, México. Líder del grupo de investigación en Administrativas, Instituto Tecnológico Metropolitano, Medellín, Colombia. Correo electrónico: juanpatino@itm.edu.co. Orcid: https://orcid.org/0000-0002-8334-9296 


\title{
Evolution and Research Trends in Marketing Oriented to Cultural and Creative Industries: a Bibliometric Analysis in Scopus
}

\begin{abstract}
The objective of this research is to examine the evolution and research trends in marketing oriented to cultural and creative industries. The methodology is based on a bibliometric analysis of 374 academic documents obtained from a search equation formulated in Scopus. For this purpose, quantity, quality and structure indicators were proposed. Among the findings, it is observed that greater focus is needed in emerging economies, since their social, cultural and economic context is particular. It is concluded that the most researched topics are: market strategy, product design, cultural factor influence, industry management (in terms of operational capabilities and operationalization) and internationalization actions aimed at the expansion of these industries.
\end{abstract}

\section{KEYWORDS}

Cultural marketing; creative industries; research trends; bibliometrics.

JEL CLASSIFICATION

C89, D83

CONTENTS

Introduction; 1. Methodology; 2. Results; Conclusions; References.

\section{Evolução e tendências de pesquisa em marketing com ênfase em indústrias culturais e criativas: uma análise bibliométrica em Scopus}

\section{RESUMO}

O objetivo desta pesquisa é examinar a evolução e tendências de pesquisa sobre marketing orientadas a indústrias culturais e criativas. A metodologia parte de uma análise bibliométrica de 374 documentos acadêmicos obtidos a partir de uma equação de busca formulada no Scopus. Para isso, foram propostos indicadores de quantidade, qualidade e estrutura. Entre os achados, observa-se que é necessária maior abordagem em economias emergentes, já que seu contexto social, cultural e econômica é particular. Conclui-se que os temas mais pesquisados são: estratégia de mercado, design de produto, influência do fator cultural, gestão da indústria (em termos de capacidades operativas e operacionalização) e ações de internacionalização orientadas à expansão dessas indústrias.

\section{PALAVRAS-CHAVE}

Marketing cultural; indústrias criativas; tendências investigativas; bibliometria.

CLASSIFICAÇÃO JEL C89, D83

\section{CONTEÚDO}

Introdução; 1. Metodologia; 2. Resultados; Conclusões; Referências. 


\section{INTRODUCCIÓN}

De acuerdo con Cuadros, Valencia y Valencia (2012) y Benita (2018), las ideas y la creatividad se han vuelto imprescindibles para cualquier economía de mercado que quiera llevar el ritmo del mundo globalizado y cambiante, por lo que no es sorpresa que la creatividad y la innovación impulsen la economía del conocimiento que surgió en la segunda década del siglo XXI y la cual, según Boix-Domenech y Soler-Marco (2017), se espera que genere estabilidad en mayor proporción en el futuro. En este sentido, para hacer alusión a aquellas industrias que fortalecen significativamente esta economía, se habla de industrias culturales y creativas.

El término fue acuñado en el Reino Unido a finales del siglo pasado por el Grupo de Trabajo de Industrias Creativas del Departamento de Cultura, Medios y Deporte (2008), se les describió como aquellas industrias cuyas actividades principales giran en torno a la creación, producción y distribución de bienes o servicios que son por naturaleza culturales y que tienden a ser protegidos por derechos de propiedad intelectual. Para Gómez-Bayona, Londoño-Montoya y Mora-González (2020), la creación de valor de las industrias se relaciona con la pertinencia de los modelos de capital intelectual en el sector. Visto desde una perspectiva más amplia, para Lampel y Germain (2016) y Herazo, Valencia y Benjumea (2017), las actividades de creatividad, habilidad y talento individual se convierten en potenciales creadoras de riqueza y empleo. Una de las características fundamentales de la cultura es la creatividad, por lo que las industrias creativas cuentan con el potencial suficiente para fortalecer la diversidad cultural, aumentar la inclusión y el desarrollo humano. Sin embargo, Potts y Cunningham (2008) mencionan que a través de la producción musical, textual, cinematográfica turística y otras, las industrias creativas consolidan su valor dinámico, en el cual contribuyen no solo a la cultura y la sociedad, sino también al crecimiento y desarrollo económico.

De acuerdo con estadísticas presentadas por la Organización de las Naciones Unidas para la educación, la Ciencia y la Cultura - Unesco (2020), estas industrias generaron 2.250.000.000 dólares estadounidenses en 2015. Estos ingresos representaron alrededor de $3 \%$ del PIB mundial, que genera más de 29.500.000 de empleos en todo el mundo. De esta manera, Wei, Chen y Hsieh (2019) afirman que la contribución de estas industrias a la economía mundial es cada vez mayor. Así, Kourtit y Nijkamp (2013) y Castiglione, Infante y Zieba (2018) encuentran que diferentes académicos alrededor del mundo han trabajado sobre los impactos en el crecimiento económico que tienen las industrias culturales y creativas, como Stam, de Jong y Marlet, (2008), Lee (2014), Cerisola (2016) y Corte-Cruz (2019), quienes analizaron el desempeño del sector en el ámbito empresarial. Así mismo, algunos 
estudios como los de Evans y Hutton (2009) y Cerisola (2016) afirman que este tipo de industrias revelan un potencial para reemplazar el empleo en declive del sector manufacturero, así como su capacidad para impulsar y fortalecer el crecimiento económico en áreas metropolitanas y atraer turistas, como lo afirman Lee (2014), Capello y Lenzi (2013) y Boix-Domenech y Soler-Marco (2017).

Por otro lado, las industrias culturales han desarrollado temas de interés específicos que se relacionan directamente con el marketing. Se han adelantado estrategias para los productos, consumidores, creativos y organizaciones en general. No obstante, estas esferas se conectan y generan mucha incertidumbre con respecto a su potencial de éxito. Peltoniemi (2015) relaciona dos elementos existentes en las industrias culturales sobre la demanda. En primer lugar, los bienes culturales son experienciales y en este sentido, los consumidores buscan novedad; en segundo lugar, casi todas las industrias culturales operan bajo rendimientos crecientes. De esta manera, el mismo autor propone explorar las relaciones entre gustos, ventas, diversidad y calidad para enfrentar la incertidumbre de los gerentes de marketing en las industrias culturales. Adicionalmente, estas industrias deben incorporar estrategias diferenciales que permitan definir indicadores claros que apunten a la medición de su competitividad como mecanismo para una planeación adecuada de su crecimiento (Acevedo-Prins y Jiménez-Gómez, 2015).

La presente bibliometría presenta la evolución y diferentes tendencias investigativas de todas las estrategias de marketing que se vuelven fundamentales para que estas industrias hagan entrega a los consumidores de ciertos productos que se detienen en elementos creativos, apuntándolos al mercado de consumo a través de la distribución masiva, en la cual las economías de escala juegan un papel de especial importancia. Estos productos, como lo menciona Benita (2018), contienen varias historias y estilos, y satisfacen las necesidades de entretenimiento y exhibición social. De la misma manera, según Lampel y Germain (2016), el marketing usado en este tipo de industrias se compone de estrategias para el intercambio de creación de valor entre las organizaciones y la sociedad mediante el uso de recursos complementarios.

Es así como el marketing engloba objetivos orientados a dejar de lado teorías tradicionales de promoción, ventas y otros aspectos, pues las organizaciones dedicadas al entretenimiento artístico y cultural, o bien aquellas que buscan posicionar un producto cultural o creativo, se ven enfrentadas a una competencia entre ellas mismas debido a la necesidad de conseguir recursos para su financiamiento. Al hacer esto último, es necesario redireccionar los productos y servicios sin pasar por alto las preferencias por segmentos de clientes para consolidar una 
estrategia en pro de la mejora de habilidades, construir audiencias propias y suplir las necesidades de los consumidores, con el fin de lograr una identificación del usuario con la empresa. Para lo anterior, juega un papel de crucial importancia la inmersión del mundo digital en las industrias creativas, dado que permite la participación creciente de los consumidores en todas las actividades de comercialización, lo cual permite mayor compromiso con las marcas. Hoy en día, de acuerdo con Avasilcai y Bujor (2018), las empresas acuerdan que para el desarrollo de productos y servicios entregados a comunidades y multitudes, las partes interesadas han de representar una fuerza impulsora para lograr el desarrollo esperado e interactivo de lo que ofrecen.

\section{METODOLOGÍA}

Según Durieux y Gevenois (2010) y Rueda, Gerdsri y Kocaoglu (2007), los análisis bibliométricos se utilizan para estudiar y medir la cantidad y calidad de libros, artículos y otras formas de publicaciones mediante métodos matemáticos y estadísticos, cuyos resultados sirven para la toma de decisiones. Además, de acuerdo con Daim et al., (2006), Ding, Chowdhury y Foo (2001) y Van Raan (2005), dicho análisis permite encontrar patrones no evidentes útiles para el avance de las investigaciones y el desarrollo científico, como también para entender el pasado y pronosticar el futuro.

Al respecto, Bordons (1999) menciona que en un estudio bibliométrico las bases de datos bibliográficas son la principal fuente de información utilizada, por lo tanto se debe elegir una que cubra el área de objeto de estudio, pues la validez de la investigación dependerá en gran medida de la base de datos seleccionada, debido a que estas difieren en cobertura temática, criterios de selección de revistas y/o documentos, sesgos geográficos y lingüísticos, entre otras características a tener en cuenta en este tipo de estudios.

En este orden de ideas, Norris y Oppenheim (2007) afirman que es de gran importancia evaluar cuál base de datos elegir para medir la producción académica, para lo cual se propusieron diferentes indicadores como: el criterio de cobertura planteado por Norris y Oppenheim (2007) y Walters (2007); el criterio de desempeño, como la cantidad de citaciones de las publicaciones sugerido por Hall (2011) y Okubo (1997); el criterio de accesibilidad planteada por Castrillón-Estrada et al., (2008) y Schroter (2006): y el criterio de flexibilidad de los operadores de búsqueda expuesta por Savoy (2005) y Walters (2007).

Según Cadavid, Awad y Cardona (2012), hoy en día dichos indicadores favorecen a bases de datos como Web of Science (ISI), Scopus y Google Scholar, por encima 
de las demás bases de datos disponibles. Sin embargo, Granda-Orive et al., (2013) afirman que Scopus, base de datos fundada por Elsevier S.L., en 2004, es el competidor más fuerte, pues se le reconocen ventajas como la facilidad de navegación y acceso a documentos citados, está abierta a internet, dispone de páginas web, patentes y enlaces a la página web de editores de revistas, así mismo, incluye el $100 \%$ de lo indexado en las bases Medline, Embase y Compedex. Además, Scopus basa sus datos de influencia en el cálculo del factor $h$, creado en un intento por medir carrera y recorrido de un autor; toma su nombre del indicador SCImago Journal Rank (SJR), el cual muestra la visibilidad de las revistas contenidas en la base de datos Scopus desde 1996.

Por lo anterior, se seleccionó la base de datos Scopus como fuente de información bibliográfica, puesto que según Boeris (2011), ofrece acceso a diferentes bases de datos interdisciplinarias, suministra herramientas para gestionar la información y cumple con otros criterios como la cantidad de citaciones y la accesibilidad, lo que la convierten en la base de datos más utilizada en este tipo de análisis de la literatura.

Posteriormente, se definió la ecuación de búsqueda, en la que se tiene en cuenta los términos principales en este estudio, que corresponden a industrias culturales, industrias creativas y marketing. En adición, estos términos se buscaron en los campos de título y palabras clave, sin incluir restricciones de tiempo con el fin de obtener los registros desde los inicios de la temática y así tener el horizonte de análisis más amplio posible. A continuación se muestra la ecuación de búsqueda construida:

(TITLE [cultural OR creative] AND TITLE [industr*] AND TITLE [marketing]) OR KEY (cultural OR creative) AND KEY (industr*) AND KEY (marketing)

Al aplicar la ecuación de búsqueda se obtuvieron 374 registros hasta el 13 de octubre del 2019, después de verificar que los registros hicieran referencia a la temática de estudio. Luego, se construyó una base de datos para analizar cada una de las variables que se incluirían en la elaboración del análisis bibliométrico. Una vez definida dicha información, se determinaron los indicadores con los cuales se realizaría el estudio, los cuales corresponden a cantidad y calidad, que miden la productividad y el impacto respectivamente. Por último, se presenta el análisis de palabras claves para la identificación de tendencias y el gráfico de evolución que muestra los enfoques realizados en los diferentes periodos de tiempo y la agenda de investigación, en la cual se mencionan diferentes enfoques para futuras investigaciones. 


\section{RESULTADOS}

\subsection{Indicadores de cantidad}

\subsubsection{Publicaciones por año}

En el gráfico 1 se puede observar la relación entre el número de publicaciones por año dentro de un periodo que comprende desde alrededor de 1980 hasta el 2019. Se puede observar que después del 2000 hay una mayor tendencia a la producción científica sobre el tema de interés, de manera que de 2003 a 2005 se da el periodo con mayor producción, se registró en el 2004 una producción superior a 35 publicaciones. También se puede observar un descenso en la producción después del 2005.

Gráfico 1. Evolución de la temática en el tiempo

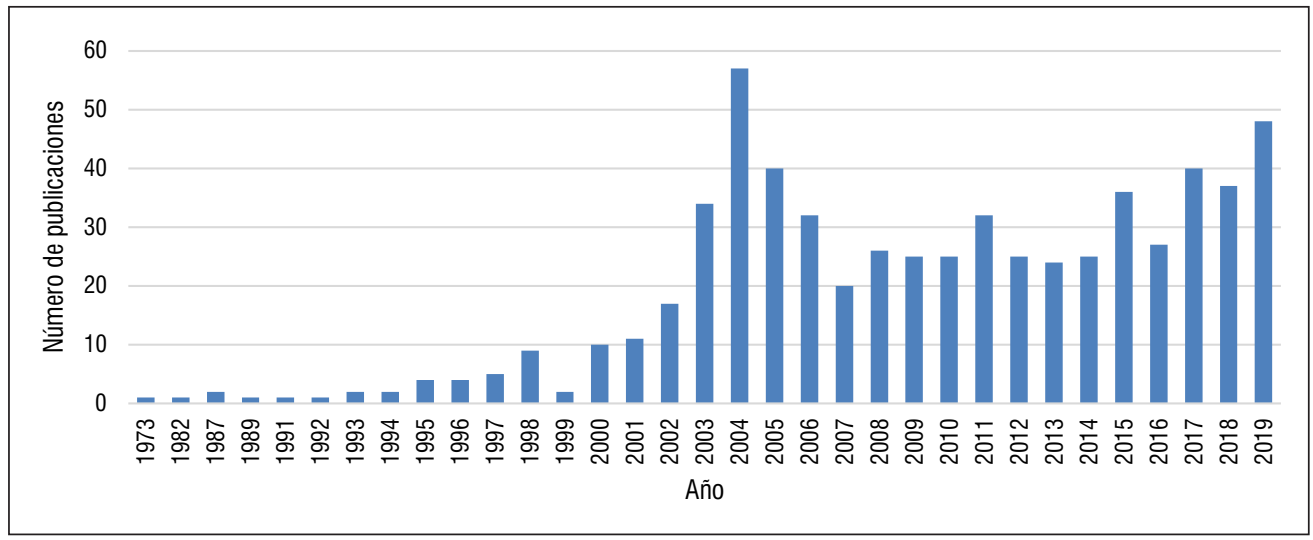

Fuente: elaboración propia obtenida a partir de los resultados de búsqueda

\subsubsection{Publicaciones acumuladas por año}

La tendencia de las publicaciones acumuladas es creciente, aun cuando el número de publicaciones a partir del 2005 disminuyó respecto a los picos de producción. Si bien el aumento en los últimos 5 años es del 4,3\% anual, se tiene un incremento del 24,5 \% anual, lo que muestra una afinidad por la investigación en este campo.

Es importante saber cuál es la vida media de la literatura que se consulta, ya que este es un dato importante para el uso de dicha información en el desarrollo de futuras investigaciones. Según Rojas-Moreno et al. (2013), la vida media de la literatura se asocia con la obsolescencia del conocimiento que está fundamentada en un periodo de tiempo en el que disminuye la utilización de la información y con ello, la vida útil de las publicaciones desarrolladas. En el gráfico 
2 se resume esta información respecto al tiempo, se puede apreciar que desde el 2005 este valor incrementa. En efecto, la vida media de todos los artículos y documentos consultados es de 6,7 años, mientras la vida media de los últimos 10 años es de 9,3 años.

\section{Gráfico 2. Vida media de la literatura}

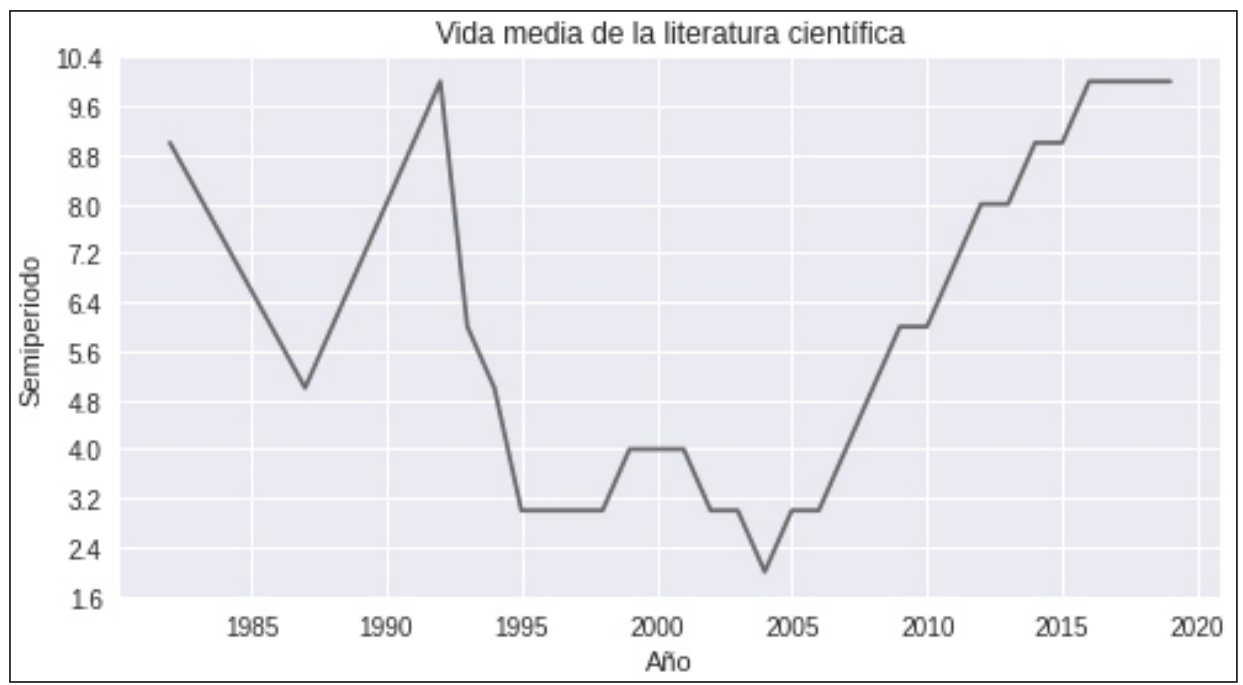

Fuente: elaboración propia obtenida a partir de los resultados de búsqueda

\subsubsection{Numero de publicaciones por revista}

El gráfico 3 presenta el listado de las diez principales revistas en las que se concentran el mayor número de publicaciones relacionadas con el mercadeo de industrias culturales y creativas. La revista que lidera este indicador es Tobacco Control con diez publicaciones, seguida por HOW y Science and Medicine con ocho publicaciones cada una. Cabe mencionar que estas revistas son especializadas en tabaco y salud, han estudiado el impacto de la publicidad realizada al tabaquismo por medio de las industrias culturales como el cine y utilizan estrategias de marketing para encontrar factores asociados a la aceptación de dicha publicidad, pues la industria del tabaco es una de las industrias que más impacto tiene con su publicidad, lo cual se ve reflejado en los altos niveles de consumo. El resto de las revistas listadas cuenta con cinco publicaciones o menos. Además, se tiene que el índice de producción refleja que el 50 \% de las publicaciones académicas sobre el tema de interés se concentra en el 35,1 \% de las revistas consultadas. De igual forma, el índice de transitoriedad muestra que el $84,5 \%$ de las revistas publicaron solamente un trabajo sobre el tema. 


\subsubsection{Distribución de publicaciones entre revistas}

El análisis de la distribución se hace con respecto a la cantidad de publicaciones entre el número de revistas obtenidas. De esta manera se tiene que el 8,9\% de las revistas publica $25 \%$ de la producción académica, el 35,1 \% de las revistas publica el $50 \%$, el $67,7 \%$ de revistas publica el $75 \%$, y el $74,2 \%$ de revistas publica el 80 $\%$ de la producción académica. Así, después de la aplicación del logaritmo natural sobre los ejes del gráfico 3 se obtiene la ley de la productividad, la cual no sigue un comportamiento lineal sino logarítmico.

Gráfico 3. Lista de las diez revistas que más publican sobre el tema

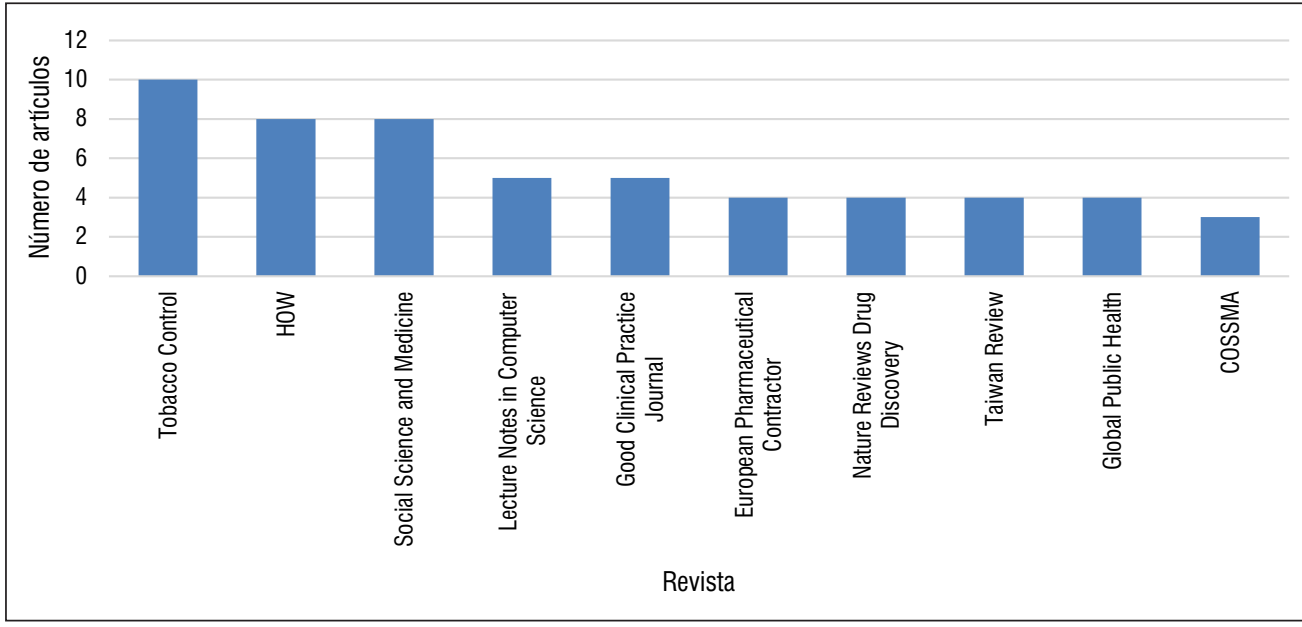

Fuente: elaboración propia obtenida a partir de los resultados de búsqueda

\subsubsection{Cantidad de publicaciones por país}

En cuanto a los países que mayores aportes hacen en producción académica en el tema de interés, Estados Unidos encabeza la lista con más de 100 publicaciones, lo cual es una cantidad de publicaciones bastante considerable, ya que en segundo lugar se encuentra el Reino Unido con 40 publicaciones. En la lista sigue China con poco más de 22 publicaciones y el resto de países con menos de 20 publicaciones.

En el gráfico 4 se puede observar el mapa con la visualización de los países y su distribución de publicaciones en la temática, en colores oscuros los países con mayor producción y en colores claros aquellos con menor producción. Esta gráfica confirma el liderazgo de los Estados Unidos, así como la distribución entre el Reino Unido como líder europeo, China en la región asiática y Australia en el continente oceánico, lo que es muestra de que el estudio sobre este tema lo han realizado países 
desarrollados, por lo tanto, surge la oportunidad de generar más investigaciones en países en desarrollo a partir de sus necesidades y particularidades culturales, sociales y económicas.

Gráfico 4. Mapa de distribución de publicaciones por país

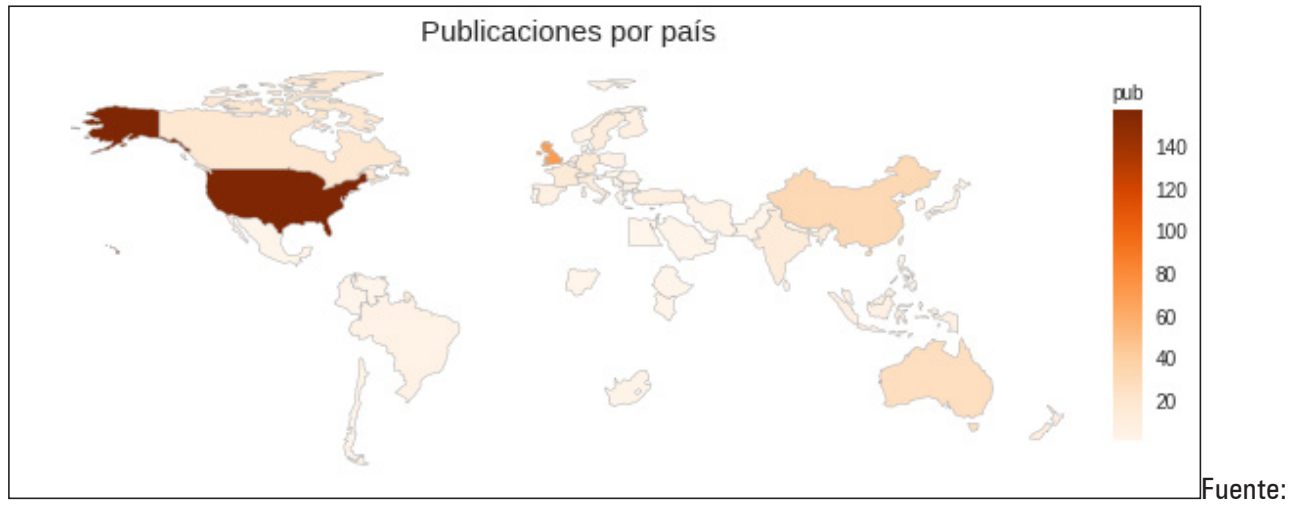

elaboración propia obtenida a partir de los resultados de búsqueda

\subsubsection{Tipo de publicaciones}

Con respecto a la distribución sobre el tipo de publicaciones que se encontraron en la búsqueda sobre el tema de interés, la mayoría de las publicaciones son tipo artículo con un 48,7 \%, seguidos de los paper de conferencias y los review (revisión o análisis, puede ser de la literatura, de investigaciones o de un libro o publicación científica) que juntos cuentan con un 36,4 \% del total de publicaciones. Los artículos muestran la madurez del tema al ser publicados en revistas especializadas, aunque se ha de resaltar la participación de los paper o actas de las conferencias, ya que son espacios en los que se hace difusión de los diferentes avances en la temática de interés.

\subsubsection{Publicaciones por autor}

Se lista a continuación a los diez autores con mayor número de publicaciones. Como se puede ver en el gráfico 5, el comportamiento está muy centrado en algunos pocos, lideran Lee, Li y Chapman, con el mismo número de publicaciones vinculadas que asciende a 30. Las publicaciones realizadas por Lee y Chapman, se relacionan con la industria del tabaco y su relación con el marketing en industrias culturales, mientras que Li, se enfoca en el estudio de estrategias para el desarrollo de industrias culturales. Muy cerca está el resto del grupo de autores y realizan 20 publicaciones cada uno, por lo que la mayor parte de la producción se concentra en este grupo. 
Gráfico 5. Numero de publicaciones por autor

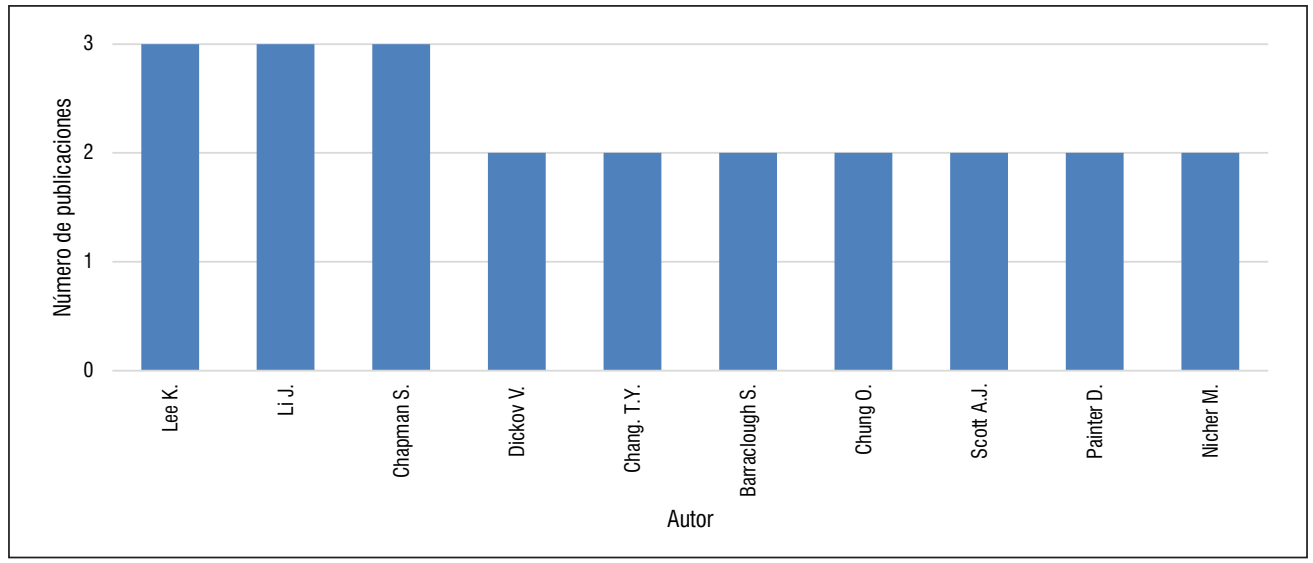

Fuente: elaboración propia obtenida a partir de los resultados de búsqueda

\subsection{Indicadores de calidad}

\subsubsection{Citaciones por revista}

Con este indicador se mide el impacto que tienen las revistas mediante la cantidad de citaciones a las publicaciones en los temas relacionados que poseen. En el gráfico 6 se pueden observar las principales revistas con el mayor número de citaciones asociadas. En primer lugar, se encuentra la revista Science que cuenta con 880 citaciones, pertenecientes al artículo "Experimental study of inequality and unpredictability in an artificial cultural market", el cual estudia los factores que determinan el éxito de productos culturales. Esta gran cantidad de citaciones se puede deber a que es una de las revistas con mayor reconocimiento en el campo académico, por lo que tiene mayor número de seguidores que acreditan su confianza en sus publicaciones. Para continuar la lista, con poco más de 240 citaciones, se encuentra la revista Urban Affairs Review que se especializa en estudios urbanos, en los que se incluye política urbana, desarrollo económico urbano y desarrollo residencial y comunitario. La publicación de esta revista corresponde a "Cultural-products industries and urban economic development: Prospects for growth and market contestation in global context" que hace referencia a la relación entre productos culturales y mercados globales. En tercer lugar, se encuentra la revista International Journal of Obesity con unas 163 citaciones que corresponden a la publicación "New insights into the field of children and adolescents' obesity: The European perspective" que plantea alternativas para tratar la obesidad en niños y adolescentes, entre las cuales se encuentra la publicidad de nutrición saludable mediante productos culturales. Luego de esta se encuentra Management Science, Proceedings of the IEEE con 157 citaciones y 
demás revistas que se listan con menor cantidad de citaciones. Cabe resaltar que ninguna de las revistas listadas se encuentra entre las que mayor producción tienen asociadas (ver gráfico 3).

Gráfico 6. Citaciones por revista

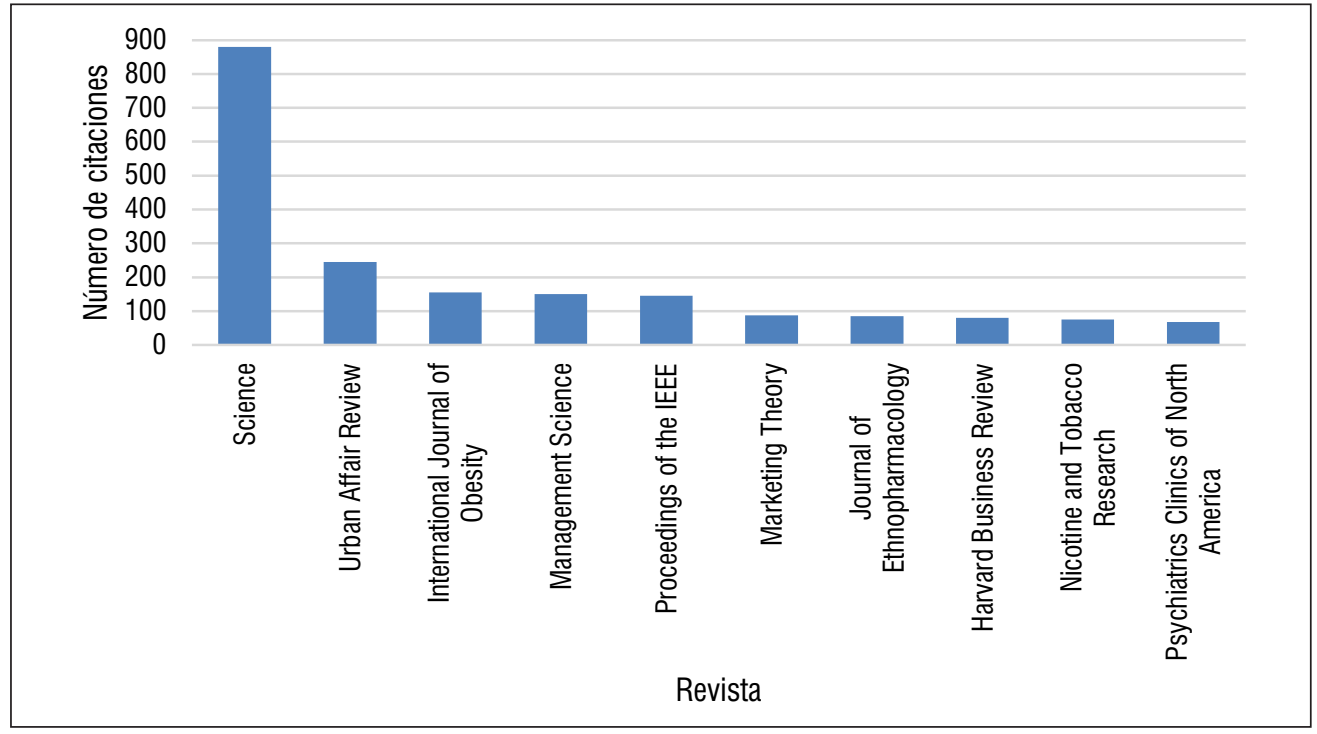

Fuente: elaboración propia obtenida a partir de los resultados de búsqueda

\subsubsection{Citaciones por países}

En este indicador se establecen las relaciones entre los países y el número de citaciones vinculadas a las publicaciones realizadas en estos países. En el gráfico 7 se pueden ver los países relacionados; encabeza la lista Italia como el país con mayor número de citaciones asociadas (53), seguido de Japón con 45 citaciones, Kenya con 44 y Hong Kong con 42. Cabe resaltar que, aunque los Estados Unidos tiene la revista Science, la cual tiene el mayor impacto por citaciones (ver gráfico 6), este el quinto país en lista con mayor impacto. El resto de los países se pueden ver en el gráfico 7.

\subsubsection{Impacto por autores}

En el gráfico 8 se resumen los diez autores que mayor impacto tienen en el tema de interés debido a que acumulan la mayor cantidad de citaciones. En primer lugar, se encuentran tres autores que tienen un trabajo sobresaliente, cada uno cuenta con alrededor de 800 citaciones asociadas, estos son Watts, Salganik y Doods. Dichas citaciones pertenecen a la publicación anteriormente mencionada, "Experimental 
study of inequality and unpredictability in an artificial cultural market" de la revista Science. Después de estos, el resto de los autores que figuran cuentan con menos de 200 citaciones, lo cual demuestra el liderazgo del trío de autores que encabezan la lista, con una diferencia muy apreciable. Los autores que siguen en la lista tienen una producción parecida en cuanto a cantidad, el autor que da cierre a la lista cuenta con 87 citaciones.

\section{Gráfico 7. Citaciones por país}

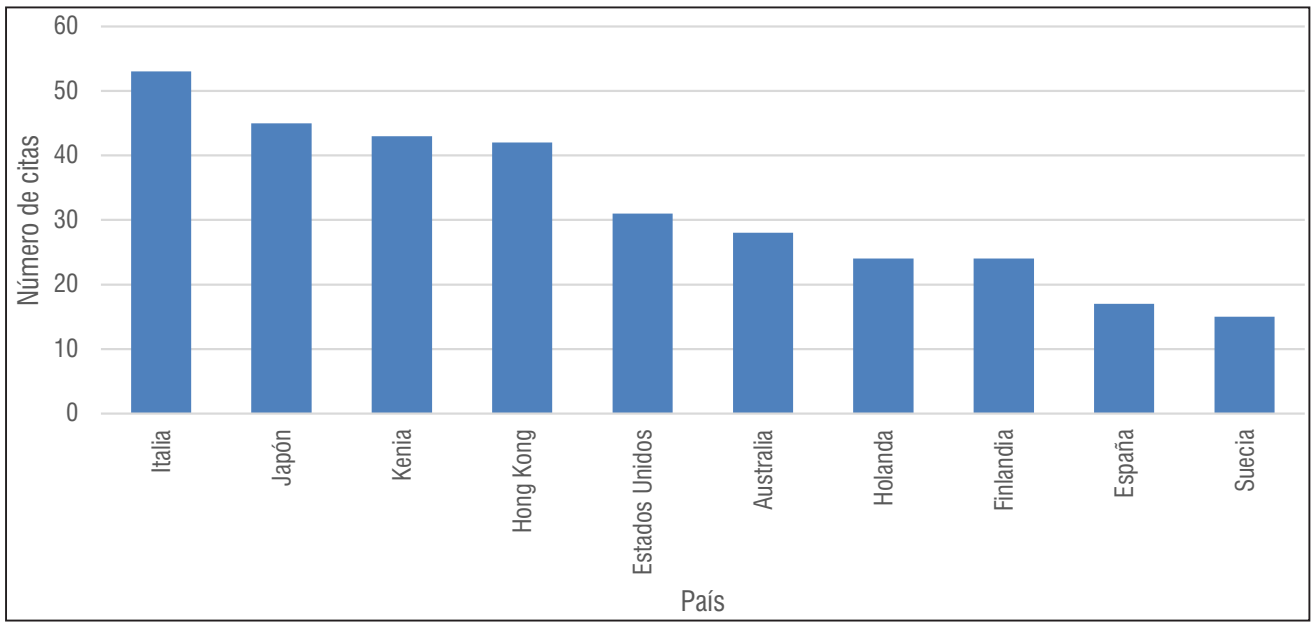

Fuente: elaboración propia obtenida a partir de los resultados de búsqueda

Gráfico 8. Impacto de autores por citaciones

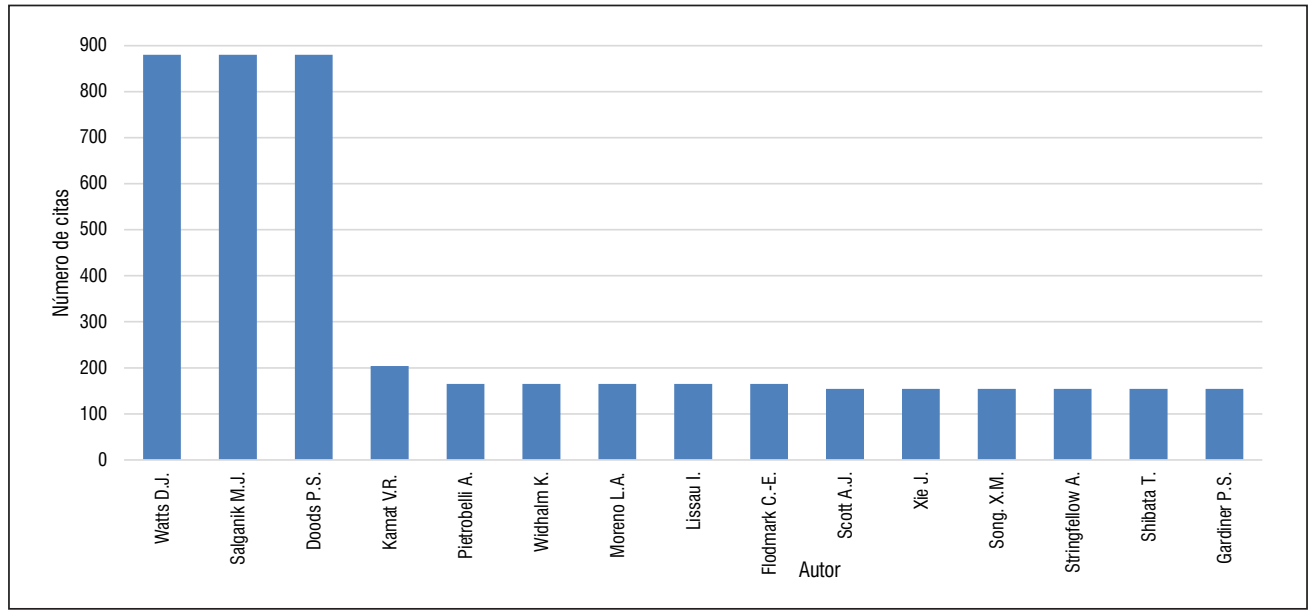

Fuente: elaboración propia obtenida a partir de los resultados de búsqueda 


\subsection{Temas emergentes y tendencias}

El gráfico 9 destaca las tendencias temáticas con respecto al mercadeo en industrias culturales y creativas a través del tiempo. Las temáticas que son tendencia y lideran el grupo están relacionadas con la comercialización de drogas, se enfoca en informar sobre el consumo responsable mediante diferentes medios en los que la industria cultural participa y el factor cultural como un aspecto que influye en la decisión de adquirir productos culturales y creativos; siguen la tendencia de género como otro factor relacionado con la adquisición de dichos productos y la industria del tabaco, pues el consumo del tabaco está influenciado por la industria cultural. Es de resaltar que de todas las tendencias de lista, ninguna es un tema emergente, sino que se han consolidado a través del tiempo.

\section{Gráfico 9. Temas emergentes y tendencias}

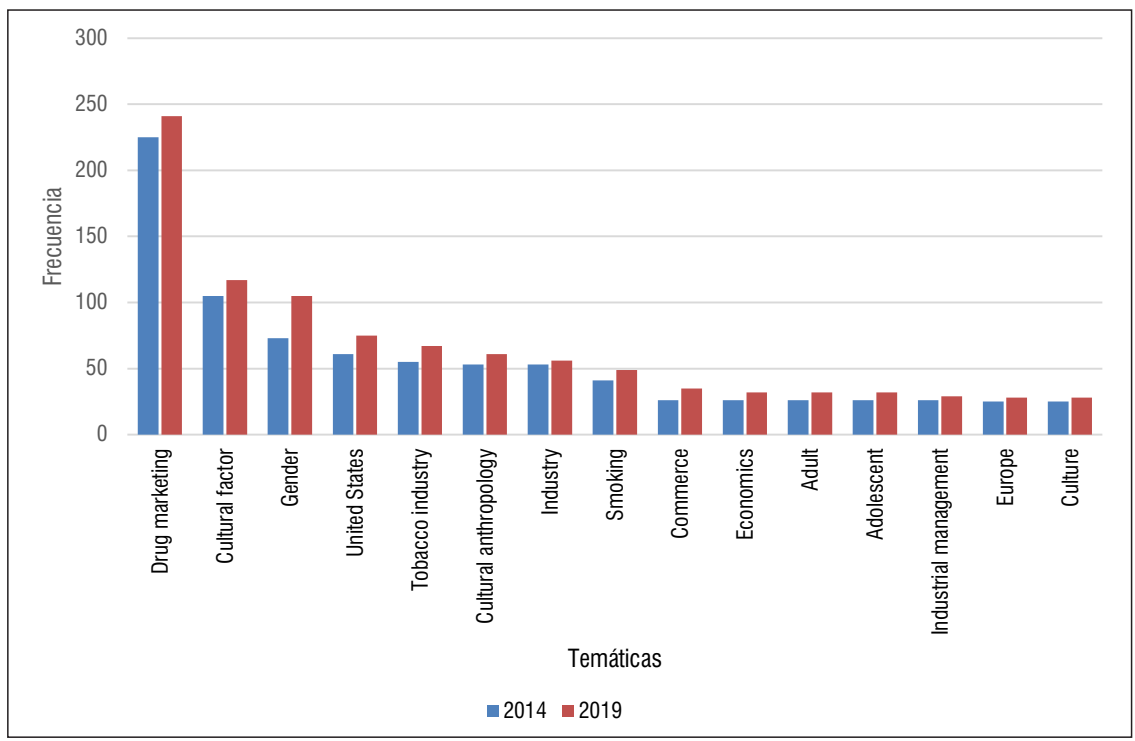

Fuente: elaboración propia obtenida a partir de los resultados de búsqueda

\subsection{Discusión de temas emergentes}

\subsubsection{Estrategia de marketing}

La industria cultural y creativa significa nuevas oportunidades de negocio, sin embargo, es importante conocer cómo atraer al público objetivo. Es por esto que Wang, Ho y Chen (2015) utilizan el marketing experimental como estrategia para estudiar la intención de compra de productos de ilustración cultural y creativa de Taiwán, en el cual los hallazgos indican que el marketing experimental puede afectar 
positivamente al marketing boca a boca y como consecuencia, aumentar la intención de compra de productos culturales y creativos. Por otra parte, Huang, Yang y Fan (2014) realizan un plan para el desarrollo de una estrategia de marketing basada en el tema de las exhibiciones y orientada por la comercialización de las industrias culturales y creativas, en este encuentran que el marketing experimental también es la mejor opción en las industrias relacionadas con las exhibiciones.

Chen, Yeh y Chu (2014) estudian la relación causal entre las estrategias de marketing, la identidad y las intenciones del consumidor hacia marcas culturales y creativas. Encuentran que los productores o comercializadores de marcas culturales podrían mejorar las relaciones consumidor-marca al promover historias culturales en exhibiciones asociadas con sus marcas. Además, para atraer visitantes, podrían despertar resonancia cultural al compartir las ideas creativas que inspiraron sus productos únicos. Además, Ku, Lo y Lin (2014) estudian el proceso de desarrollo de la industria cultural y creativa de tallado en madera y proponen diferentes estrategias de marketing, por ejemplo el marketing emocional, con el fin de promover la participación proactiva y la compra de productos de tallado en madera, debido a que, aunque esta industria presentó un apogeo en sus exportaciones y ventas nacionales en Taiwan, en la actualidad y por diferentes cambios en el ciclo económico, ha presentado un decrecimiento en su comercialización.

\subsubsection{Diseño de producto}

Avasilcai y Bujor (2018) mencionan que el mundo digital desempeña un papel importante en la actualidad, como lo demuestran las investigaciones recientes sobre marketing digital. Por lo tanto, en el 2000 se creó la comunidad en línea de artistas Threadless, la cual busca nuevos productos y diseños que se puedan ofrecer a mentalidades diferentes y creativas. Así, la investigación mostró -por medio del estudio de la plataforma Threadless- que el diseño en el desarrollo de nuevos productos como fuente de innovación abierta en las industrias creativas es de gran importancia, pues contribuye a que estos tengan mayor aceptación en el mercado.

Con el fin de desarrollar productos competitivos para hacer frente a un entorno cambiante y de competencia en la industria del turismo y con el objetivo de hacer turismo cultural, Wang (2018) menciona que el marketing interactivo juega un papel importante, ya que los resultados de su estudio arrojan que este presenta correlaciones significativamente positivas con el retorno de la inversión, la excelencia en el servicio, la estética y la alegría. Por lo tanto, la implementación del marketing interactivo como parte del diseño en el desarrollo de los nuevos productos culturales hace que estos presenten mayor competitividad. Dichos resultados fueron útiles 
para proponer opciones que ayuden a las empresas de turismo cultural para crear valor por medio de sus productos.

Por otra parte, Chang (2014) menciona que debido a los problemas actuales en el proceso de producción de mercancías culturales, incluidos los contextos de creatividad, productividad de las tendencias de la moda y funciones adicionales, a menudo faltan las bases específicas para la evaluación de los diseños de mercancías culturales. Como resultado, los trabajos de los diseñadores no tienen eco ni reciben reconocimiento de los consumidores, lo que conduce a dificultades en las ventas. Por lo tanto, en este artículo se encontró que es necesario implementar un modelo de comercialización y marketing dentro del diseño de los productos, tal que les permita la participación en mercados modernos y una mayor aceptación.

\subsubsection{Factor cultural}

El marketing también se ha utilizado para estudiar las diferentes culturas en el turismo con el fin de saber qué es un servicio de calidad para cada una de ellas a partir del país de origen. Un ejemplo de esto es la publicación de Li y Han (2009) que identifica que la dimensión cultural se relaciona con la calidad del servicio y con la intención de tomarlo. Por lo tanto, en dicho estudio se propone desarrollar diferentes estrategias de marketing con el fin de hacer atractivos los servicios de turismo a todos los tipos de turistas. Otro estudio que muestra la influencia de la cultura lo conducen Margariti et al., (2018), por medio de la publicidad de alimentos, en específico los anuncios impresos para un nuevo café y una nueva marca de helados, encuentran que en la cultura de la India dichas metáforas visuales parecen tener un efecto más positivo en la actitud hacia el anuncio y finalmente, en la actitud hacia la marca en comparación con los contextos de una cultura estadounidense.

Por otra parte, en el estudio realizado por Ivanov e Ivanova (2016) se mide el impacto de la cultura en el nivel de penetración en el mercado de la industria hotelera y se encuentra que el nivel de penetración en dicho mercado es mayor en países con puntaje alto en individualismo cultural. La investigación de Viet Ngo y O'Cass (2011) hace una contribución a la literatura de orientación empresarial al demostrar la utilidad de un enfoque de comportamiento cultural en la medición de la orientación del marketing y la orientación a la innovación como contribuidores a la creación de valor centrado en el cliente en diferentes entornos empresariales.

\subsubsection{Comercio internacional}

Cheng, Huang y Su (2013) estudian cómo la industria de animación en China ingresó en el mercado internacional y discuten diferentes estrategias de marketing 
como la construcción de bibliotecas de animación chinas en diferentes lugares en el extranjero, con el fin de implementar la comunicación de marketing de imágenes de animación chinas, expandir los grupos de consumidores, obtener información de la audiencia y explorar el mercado internacional. Luego, presentan iniciativas estratégicas específicas con la esperanza de proporcionar algunas ideas nuevas para las estrategias de internacionalización de la industria de la animación china.

Como se mencionó anteriormente sobre el aspecto cultural, Li y Han (2009) proponen que se utilice el marketing para que el turismo, en un lugar específico, sea atractivo para personas de diferentes países y culturas, con lo que se lograría aumentar la cantidad de visitantes en dicho lugar. Por otra parte, el éxito mundial de la música es visto por Erbacher y Nitzsche (2017) como una oportunidad para cambiar la imagen del lugar de origen y de sus comunidades sociales y culturales. Así también, hacen uso del marketing para mostrar dichos lugares, lo cual ayuda a establecer una identidad postindustrial nueva, inclusiva y segura, además de contribuir a una imagen urbana más positiva en el mundo.

Otro estudio que analiza el comercio internacional lo realiza Cai (2012), menciona que el comercio de los productos de piedra tiene un valor agregado bajo y la competencia de precios también es muy feroz, sin embargo, debido a las características inherentes a la piedra, su valor agregado puede aumentar considerablemente al extender su funcionalidad a través del diseño de productos culturales y creativos, como también por medio del brand marketing, el cual puede expandir su volumen y territorio de ventas.

\subsection{Evolución del marketing orientado a industrias culturales y creativas}

En el gráfico 10 se presenta el resumen de la evolución, el cual muestra los aspectos estudiados a lo largo del tiempo con respecto al tema de marketing orientado a industrias culturales y creativas. Se observa que para los primeros años de estudio sobre el tema (1990-2000), el enfoque busca mostrar cómo por medio de la creatividad las industrias agregan valor a su proceso, también algunos autores se enfocan en promover el uso de las TIC e I+D como estrategias corporativas. Entre 2001 y 2005 el estudio sobre el tema empezó a tener un crecimiento significativo, se observa el análisis del ámbito legal que permite mayor comercialización de productos creativos y culturales en el ámbito nacional e internacional. En este periodo de tiempo también se estudiaron los factores que permiten tener más creatividad y por ende más éxito en la industria en la que se desempeña cada organización. Se encuentra también que ciertos lugares tienen un mayor número de industrias creativas que otros, lo cual se debe a aspectos como la innovación, apoyo a las empresas, cultura, entre otros. 
Para el siguiente periodo (2006-2010), el enfoque se relaciona con el desarrollo de nuevos productos apoyado en herramientas tecnológicas y la importancia que tiene que dichos productos sean de calidad para sobresalir en el mercado y ser atractivos. También se realizan estudios sobre la administración de las diferentes técnicas de marketing y la importancia que tienen los comentarios de expertos en cuanto a productos culturales para tomar la decisión de adquirirlos o no. Para el periodo comprendido entre el 2011 y el 2015, diferentes estudios analizan productos cinematográficos, musicales y de turismo, en los cuales se hacen relaciones entre estos y variados tipos de marketing que hacen que dichos productos sean atractivos en el mercado o generen otro tipo de impactos como el cambio de imagen de los lugares desde los cuales son originarios. Por otra parte, se estudian los factores que influyen en el comportamiento de compra de los productos creativos y culturales y se muestra la importancia de construir una marca para efectos de mercadeo como la recordación, entre otros.

\section{Gráfico 10. Gráfico de la evolución temática}

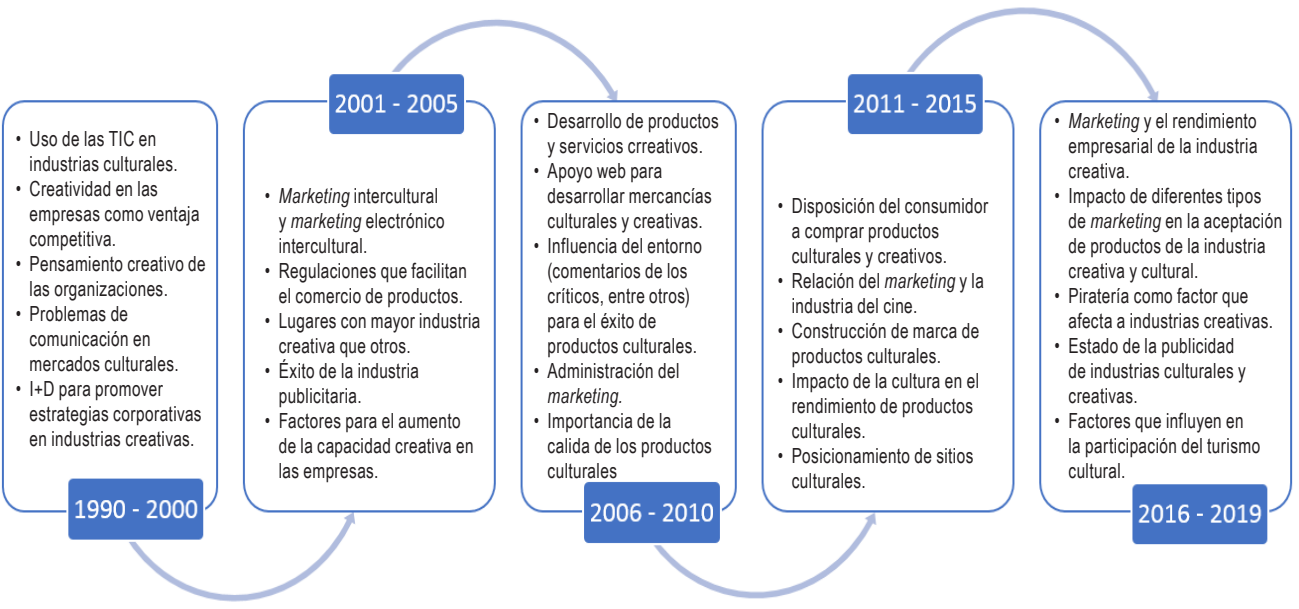

Fuente: elaboración propia

Por último, para el periodo entre 2016 y 2019 se abordan aspectos como la relación del marketing con el rendimiento de una empresa, que, por lo que arrojan los resultados, no afecta significativamente. También se analiza de nuevo el impacto de diferentes tipos de marketing -en su mayoría marketing verde-en el impacto sobre la aceptación de productos culturales y creativos. Adicionalmente se estudia cómo la piratería en industrias culturales tiene repercusiones negativas, pues hace que disminuyan las inversiones para la producción. Por último, se estudian los factores 
que influyen en la participación del turismo cultural y se encuentra que la percepción de afectar el ecosistema ocasiona que se evite tomar el servicio, por lo tanto, este es un caso en el que el marketing verde se puede aplicar.

\subsection{Agenda de investigación}

Con el fin de dar una orientación a futuras investigaciones relacionadas con marketing en industrias culturales y creativas, a continuación, se presenta una agenda formativa en la cual se enuncian temas relevantes para su estudio, a partir de los hallazgos del presente análisis bibliométrico.

- Con respecto a la participación de los países incluidos en la investigación sobre el tema, se observa que en los países desarrollados se encuentra el mayor número de publicaciones, por lo tanto, se presenta la oportunidad de investigar sobre el tema en los países en desarrollo a partir de las necesidades y particularidades culturales, sociales y económicas.

- Debido a que se observa una disminución en el estudio del marketing en industrias culturales y creativas desde el 2005 -lo cual puede ser causado por diferentes aspectos, entre los que se encuentra el estudio de factores específicos y no del tema en general como se abarca en este análisis-, otra oportunidad de estudio que podría ser de gran interés está relacionado con investigar cuáles son los temas que ya se han estudiado sobre el marketing en industrias culturales y creativas de manera específica y que por dicha razón ya no se incluyen en este estudio.

- A partir de lo mencionado por Lee y Watters (2016), se observa que la piratería es un factor que afecta en gran medida a las industrias culturales y creativas, pues hace que disminuya la rentabilidad de los productos y por lo tanto las inversiones en estos. Sin embargo, es un aspecto que ha sido poco abordado o relacionado con el tema en estudio. Por lo tanto, evaluar este factor sería de gran importancia para identificar qué técnicas se han desarrollado para atacar dicho problema y/o qué nuevas propuestas pueden surgir.

- Diferentes autores como Li y Han (2009), Margariti et al., (2018) e Ivanov e Ivanova (2016), mencionaron que el factor cultural y el género influyen en la decisión de adquirir productos culturales y creativos, sin embargo, no hay información suficiente sobre qué otros factores pueden influir en dicha decisión. Es así que otra oportunidad de estudio dentro del tema es investigar los factores asociados con la intención de compra de dichos productos. 


\section{CONCLUSIONES}

Las industrias creativas y culturales surgen a partir del talento individual, con fines de entretenimiento y creación de valor cultural en la sociedad. Por ello, el marketing en este tipo de industrias se diferencia del marketing tradicional, sobre todo en cuanto a las estrategias de promoción y ventas, pues permite a las organizaciones que se dedican al entretenimiento artístico y cultural sacar ventajas y ganancias con la puesta en marcha de estrategias innovadoras y más eficaces a través del conocimiento de la audiencia.

El marketing en industrias creativas y culturales es un tema que ha cobrado especial importancia en la última década y el cual debe ser visto de manera holística dada la urgente necesidad de posicionamiento exigida por la alta competitividad de la industria. Por ello, la estrategia de marketing debe englobar objetivos orientados a la inclusión de los segmentos de mercado en la construcción de contenido y comunidad y de este modo, consolidar audiencias que propicien crecimiento y estabilidad en el tiempo a las organizaciones.

Un hallazgo importante de la revisión de la literatura se relaciona con el factor cultural, se encontró que en las industrias culturales y creativas este afecta en gran proporción el interés de las personas por adquirir un producto. Es decir, las preferencias se ven afectadas por factores como la nacionalidad y las costumbres, por lo que se vuelve necesario identificar el tipo de público objetivo, su procedencia y comportamiento, con el fin de lograr por medio del marketing, que dichos productos y servicios sean atractivos para ellos.

Diferentes autores como Lumbaraja, Lubis, y Hasibuan (2019) y Santoso, Ariqoh y Maulida (2019), ven en el marketing verde una oportunidad para hacer atractivo el turismo de ciertos lugares, pues encuentran que por medio de este, los turistas perciben que el territorio visitado no se ve afectado por sus actividades. Este hallazgo puede significar para la industria del turismo una oportunidad de negocio que haga más atractivo su servicio y de esta forma ampliar su público objetivo, que incluiría entonces a aquellas personas que buscan proteger el ecosistema.

A partir del análisis de tendencias se observa que a lo largo del tiempo se han asociado nuevos factores que influyen en la creación de servicios creativos y culturales, como lo son el uso de tecnologías de información, los medios publicitarios, diferentes tipos de marketing y el estudio de características como el país de origen y la cultura del mercado objetivo, los cuales permiten una mayor aceptación de dichos productos o servicios.

Para los últimos años, aunque se ha observado un descenso en el número de publicaciones sobre el tema, existe un mayor interés por relacionar diferentes tipos 
de marketing con productos y servicios culturales y creativos, lo cual permite obtener resultados cada vez más acertados en cuanto a la aceptación de estos por parte del público objetivo, para lograr así el aumento del mercado en dicha industria, pues los productos aumentan su reconocimiento y poder de ser elegidos.

\section{REFERENCIAS}

Acevedo-Prins, Natalia y Jiménez-Gómez, Luis (2015). Índice para la medición de la competitividad en Colombia. En: Revista CEA, Vol. 1, №. 2, p. 109-121. Doi: 10.22430/24223182.136

Avasilcai, Silvia y Bujor, Adriana (2018). Open innovation in creative industries. Part II: The case of Threadless. En: IOP Conference Series: Materials Science and Engineering, Vol. 400, №. 6. Doi: $10.1088 / 1757-899 X / 400 / 6 / 062002$

Benita, Francisco (2018). On the performance of creative industries: Evidence from Mexican metropolitan areas. En: Papers in Regional Science, Vol. 98, N. 2, p. 825-842. Doi: 10.1111/ pirs.12403

Boeris, Claudia (2011). Las fuentes de datos en los estudios bibliométricos. En: II Jornada de Intercambio y Reflexión acerca de la Investigación en Bibliotecología. La Plata, Argentina: Universidad Nacional de la Plata, 13p.

Boix-Domenech, Rafael y Soler-Marco, Vicent (2017). Creative service industries and regional productivity. En: Papers in Regional Science, Vol. 96, №. 2, p. 261-279. Doi: 10.1111/pirs.12187

Bordons, María (1999). Evaluación de la actividad científica a través de indicadores bibliométricos. En: Revista Española de Cardiología, Vol. 52, №. 10, p. 790-800. Doi: 10.1016/ S0300-8932(99)75008-6

Cadavid, Lorena; Awad, Gabriel y Cardona, Carlos Jaime (2012). Análisis bibliométrico del campo modelado de difusión de innovaciones. En: Estudios Gerenciales: Journal of Management and Economics for Iberoamerica, Vol. 28, $\overline{\mathrm{N}}^{\circ}$. extraordinario 0 (edición especial). p. 213-236. Doi: 10.18046/j.estger.2012.1486

Cai, Hui Wei (2012). The Creative Design of Stone and its International Marketing. Applied Mechanics and Materials. En: Trans Tech Publications, Vol. 209-211 p. 215-220. Doi: 10.4028/ www.scientific.net/AMM.209-211.215

Capello, Roberta y Lenzi, Camilla (2013). Territorial patterns of innovation: a taxonomy of innovative regions in Europe. En: The Annals of Regional Science, Vol. 51, №. 1, p. 119-154. Doi: $10.1007 / \mathrm{s} 00168-012-0539 \overline{-8}$

Castiglione, Concetta; Infante, Davide y Zieba, Marta (2018). Technical efficiency in the Italian performing arts companies. En: Small Business Economics, Vol. 51, N. 3, p. 609-638. Doi: 10.1007/s11187-017-9931 1

Castrillón-Estrada, Jaime; García-Domínguez, Juan Camilo; Anaya-Taboada, Marco; Rodríguez-Berdugo, Deisy; De la Rosa-Barranco, Dereck y Caballero-Uribe, Carlo (2008). Bases de datos, motores de búsqueda e índices temáticos: herramientas fundamentales para el ejercicio médico. En: Revista Salud Uninorte, Vol. 24, Nº. 1, p. 96-119. 
Cerisola, Silvia (2016). Creativity and local economic development: The role of synergy among different talents. En: Papers in Regional Science, Vol. 97, N. 2, p. 199-215. Doi: 10.1111/ pirs. 12254

Chang, Tsen-Yao (2014). Co-creative Value for Cultural and Creative Economic Growth-Designing a Cultural Merchandise and Constructing a Marketing Model. En: International Conference on Cross-Cultural Design. Springer, Cham, Vol. 8528 p. 427 $\overline{436}$. Doi: 10.1007/978-3-319 07308-8_ 41

Cheng, Yanfei; Huang, Sifan y Su, Feng (2013). Study on the overseas communication strategies of Chinese animation products under the background of cultural export-With building overseas Chinese animation library as an example. En: 6th International Conference on Information Management, Innovation Management and Industrial Engineering, Vol. 3, p. 243-246. Doi: 10.1109/ICIII.2013.6703560

Chen, Tser-Yieth; Yeh, Tsai-Lien y Chu, Chia-Hui (2014). Storytelling and Brand Identity in Cultural Digital Archives Industry. En: International Journal of Information and Management Sciences, Vol. 25, №. 2, p. 157-179. Doi: 10.6186/IJIMS.2014.25.2.5

Corte-Cruz, Pablo Sigifrido (2019). La demanda agregada de la cultura en México: propensión al consumo y tasa de interés para empresas culturales. En: Revista CEA, Vol. 5, No. 10, p. 151-165. DOI: $10.22430 / 24223182.1331$

Cuadros, Jonatan, Valencia, Jackeline y Valencia, Alejandro (2012). Creatividad: concepciones estratégicas y su estimulación en entornos educativos. En: Revista de Educación y Desarrollo Social, Vol. 6, N. 2, p. 138-153.

Daim, Tugrul; Rueda, Guillermo; Martin, Hilary y Gerdsri, Pisek (2006). Forecasting emerging technologies: Use of bibliometrics and patent analysis. En: Technological Forecasting and Social Change, Vol. 73, N. 8, p. 981-1012. DOI: 10.1016/j.techfore.2006.04.004

Departamento de Cultura, Medios y Deporte (2008). Creative Britain, Reino Unido: New Talents for the New Economy, p. 77.

Ding, Ying; Chowdhury, Gobinda y Foo, Schubert (2001). Bibliometric cartography of information retrieval research by using co-word analysis. En: Information Processing $\&$ Management, Vol. 37, No. 6, p. 817-842. DOI: 10.1016/S0306- $\overline{4573(00) 00051-0}$

Durieux, Valérie y Gevenois, Pierre Alain (2010). Bibliometric indicators: quality measurements of scientific publication. En: Radiology, Vol. 255, №. 2, p. 342-351. Doi: 10.1148/radiol.09090626

Erbacher, Eric y Nitzsche, Sina (2017). Performing the double rupture: Kraftklub, popular music and post-socialist urban identity in Chemnitz, Germany. En: International Journal of Cultural Studies, Vol. 20, N. 4, p. 437-455. DOI: 10.1177/1367877916638730

Evans, Graeme y Thomas, Hutton (2009). Creative cities, creative spaces and urban policy. En: Urban Studies, Vol. 46, N. 5-6, p. 1003-1040. DOI: 10.1177/0042098009103853

Gómez-Bayona, Ledy, Londoño-Montoya, Erica, y Mora-González, Beatriz (2020). Modelos de capital intelectual a nivel empresarial y su aporte en la creación de valor. En: Revista CEA, Vol. 6, Nº. 11, p. 165-184. DOI: 10.22430/24223182.1434 
Evolución y tendencias investigativas en marketing orientado a industrias culturales y creativas: un análisis bibliométrico en scopus

Granda-Orive, José Ignacio; Alonso-Arroyo, Adolfo; García-Río, Francisco; Solano-Reina, Seguismundo; Jimenez-Ruiz, Carlos Andrés y Aleixandre-Benavent, Rafael (2013). Ciertas ventajas de Scopus sobre Web of Science en un análisis bibliométrico sobre tabaquismo. En: Revista Española de Documentación Científica, Vol. 36, Nº. 2, p. 011. DOI: 10.3989/redc. 2013.2 .941

Hall, Michael (2011). Publish and perish? Bibliometric analysis, journal ranking and the assessment of research quality in tourism. En: Tourism Management, Vol. 32, Nº 1, p. 16-27. DOI: 10.1016/j.tourman.2010.07.001

Herazo, Carolina, Valencia, Alejandro y Benjumea, Martha. (2017). Perspectivas investigativas en el estudio de las industrias culturales y creativas. En: Revista KEPES, Vol. 15, №. 17, p. 24-66.

Huang, Ya-Ling; Yang, Shu-Wen y Fan, Hsu (2014). To view interdisciplinary integration of cultural and creative industries and the experiential marketing strategy from the point of feature exhibitions -taking the exhibition experience of The Delight of Chinese Character Festival as an example. En: Innovation in Design, Communication and Engineering: Proceedings of the 2014 3rd International Conference on Innovation, Communication and Engineering (ICICE 2014), Guiyang, Guizhou, PR China, p. 249.

Ivanov, Stanislav Hristov y Ivanova, Maya (2016). Market penetration of hotel chains: Does culture matter?. En: Annals of Tourism Research, Vol. 57, Nº. C, p. 259-264. DOI: 10.1016/j. annals.2015.11.021

Kourtit, Karima y Nijkamp, Peter (2013). In search of creative champions in high $\square$ tech spaces: a spatial application of strategic performance management. En: Journal of regional science, Vol. 53, N. 5, p. 749-777. DOI: 10.1111/jors.12087

Ku, Meei Yuh; Lo, Kui Hsing y Lin, Wen Tsann (2014). An Exploration of Success Factors for the Comprehensive Construction of a Shopping Area from the Consumer Point of View -A Case Study of the Business Area of Sanyi, Miaoli. En: Applied Mechanics and Materials. Trans

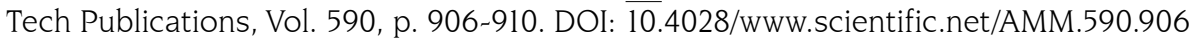

Lampel, Joseph y Germain, Olivier (2016). Creative industries as hubs of new organizational and business practices. En: Journal of Business Research, Vol. 69, №. 7, p. 2327-2333. DOI: 10.1016/j.jbusres.2015.10.001

Lee, Neil (2014). The creative industries and urban economic growth in the UK. En: Environment and Planning A, Vol. 46, N. 2, p.455-470. DOI: 10.1068/a4472

Lee, Seung Jun y Watters, Paul (2016). Gathering intelligence on high-risk advertising and film piracy: A study of the digital underground. En: Automating Open Source intelligence, p. 89-102. DOI: 10.1016/B978-0-12-802916-9.00006-3

Li, Zhihui y Han, Jinsoo (2009). A cross-cultural analysis of customer perception towards hotel service quality and behavioral intention. En: 2009 6th International Conference on Service Systems and Service Management. IEEE, Vol. 1, p. 700-703. DOI: 10.1109/ ICSSSM.2009.5174971

Lumbaraja, Prihatin; Lubis, Arlina Nurbaity y Hasibuan, Beby Kendida (2019). Sustaining Lake Toba's Tourism: Role of Creative Industry, Green Tourism Marketing and Tourism Experience. En: AJBA, Vol. 12, N. 1, p. 257-278. DOI: 10.22452/ajba.vol12nol.9 
Margariti, Kostoula; Boutsouki, Christina; Hatzithomas, Leonidas y Zotos, Yorgos. (2018). Visual metaphors in food advertising: A cross-cultural study. En: Food Research International, Vol. 115, p. 338-351. DOI: 10.1016/j.foodres.2018.11.030

Norris, Michael y Oppenheim, Charles (2007). Comparing alternatives to the Web of Science for coverage of the social sciences' literature. En: Journal of Informetrics, Vol. 1, No. 2, p. 161-169. DOI: 10.1016/j.joi.2006.12.001

Okubo, Yoshiko (1997). Bibliometric Indicators and Analysis of Research Systems, Paris, Francia: Methods and Examples, 70p.

Peltoniemi, Mirva (2015). Cultural industries: Product-market characteristics, management challenges and industry dynamics. En: International Journal of Management Reviews, Vol. 17, No. 1, p. 41-68. DOI: $10.1111 /$ ijmr. $\overline{12036}$

Potts, Jason y Cunningham, Stuart (2008). Four models of the creative industries. En: International journal of cultural policy, Vol. 14, N. 3, p. 233-247. DOI: 10.1080/10286630 $\overline{80} 2281780$

Rojas-Moreno, Bexy, Cásares, Marjorie, Viloria, Francisca y Chaparro-Martínez, Exio (2013). Análisis Bibliométrico de la Literatura Científica Publicada en la Revista de la Facultad de Ciencias Veterinarias de la Universidad Central de Venezuela. Período 2002-2011. Indicadores de Consumo. En: Revista de la Facultad de Ciencias Veterinarias, Vol. 54, Nº. 2, p. 116-126.

Rueda, Guillermo; Gerdsri, Pisek y Kocaoglu, Dundar (2007). Bibliometrics and social network analysis of the nanotechnology field. En: PICMET'07-2007 Portland International Conference on Management of Engineering $\bar{E}$ Technology. IEEE. p. 2905-2911. DOI: 10.1109/ PICMET.2007.4349633

Santoso, Imam., Ariqoh, T., y Maulida, N. (2019). Green marketing strategy for local specialty agro-industry development to support creative agro-industry. En: IOP Conference Series: Earth and Environmental Science, Vol. 230, N. 1, p. 12-52. DOI: 10.1088/1755-1315/230/1/012052

Savoy, Jacques (2005). Bibliographic database access using free-text and controlled vocabulary: an evaluation. En: Information Processing \& Management, Vol. 41, N. 4, p. 873-890. DOI: 10.1016/j.ipm. 2004.01 .004

Schroter, Sara (2006). Importance of free access to research articles on decision to submit to the BMJ: survey of authors. En: BMJ, Vol. 332, N. 7538, p. 394-396. DOI: 10.1136/ bmj.38705.490961.55

Stam, Erik; De Jong, Jeroen Pj y Marlet, Gerard (2008). Creative industries in the Netherlands: Structure, development, innovativeness and effects on urban growth. En: Geografiska Annaler: Series B, Human Geography, Vol 90, N. 2, p. 119-132. DOI: 10.1111/j.1468-0467.2008.00282.x

Van Raan, Anthony (2005). Fatal attraction: Conceptual and methodological problems in the ranking of universities by bibliometric methods. En: Scientometrics, Vol. 62, N 1, p. 133-143. DOI: 10.1007/s11192-005-0008-6

Viet Ngo, Liem y O'cass, Aron (2011). The relationship between business orientations and brand performance: A cross-national perspective. En: Asia Pacific Journal of Marketing and Logistics, Vol. 23, N. 5, p. 684-713. DOI: 10.1108/13555851111183093 
Unesco (2020). Data for the Sustainable Development Goals. París, Francia: Unesco.

Walters, William (2007). Google Scholar coverage of a multidisciplinary field. En: Information Processing E Management, Vol. 43, Nº. 4, p. 1121-1132. DOI: 10.1016/j.ipm. $\overline{2006.08 .006}$

Wang, Shu-Mei (2018). Effects of interactive marketing on value co-creation in cultural tourism. En: Journal of Interdisciplinary Mathematics, Vol. 21, №. 2, p. 489-497. DOI: $10.1080 / 0 \overline{97} 20502.2017 .1420578$

Wang, Yu-Bing; Ho, Ching-Wie y Chen, Tina (2015). Does sense of virtual community and experience marketing increase the purchase intention of cultural and creative illustration products?. En: 2015 8th International Conference on Ubi-Media Computing (UMEDIA). IEEE, p. 350-354. DOI: 10.1109/UMEDIA.2015.7297484

Wei, Mingchw; Chen, Tzuhan y Hsieh, Yungcheng (2019). Study of Cultural Creative Merchandises of Museums and Cultural Heritage. En: Advances in Graphic Communication, Printing and Packaging. Springer, Singapore, Vol. 543, p. 447-451. DOI: 10.1007/978-981-13-3663-8_60 